\title{
Laboratory and demographic findings among patients with coronavirus disease 2019: a review
}

\author{
Atefeh Mir ${ }^{1}$, Khadijeh Kalan Farmanfarma ${ }^{2}$, Somayeh Heidarpour Kiasara ${ }^{3}$, Hamid Salehiniya ${ }^{4}$, \\ Abolfazl Shakiba ${ }^{5}$, Neda Mahdavifar6
}

${ }^{1}$ Clinical Research Center of Sabzevar Vasei Hospital, Sabzevar University of Medical Sciences, Sabzevar; ${ }^{2}$ Department of Epidemiology, Health Promotion Research Center, Zahedan University of Medical Sciences, Zahedan; ${ }^{3}$ Department of Anesthesia, Zahedan University of Medical Sciences, Zahedan; ${ }^{4}$ Social Determinants of Health Research Center, Birjand University of Medical Sciences, Birjand; ${ }^{5}$ Department of Internal Medicine, School of Medicine, Leishmaniasis Research Center, Vasei Hospital, Sabzevar University of Medical Sciences, Sabzevar; ${ }^{6}$ Department of Biostatistics and Epidemiology, School of Health, Non-Communicable Diseases Research Center, Sabzevar University of Medical Sciences, Sabzevar, Iran

\begin{abstract}
Coronavirus disease 2019 (COVID-19) is the third known animal coronavirus, after severe acute respiratory syndrome (SARS) and Middle East respiratory syndrome coronavirus (MERS-CoV). The mean age of the infected patients was estimated to be between 50 and 69 years old. Accordingly, the COVID-19 mortality rate was calculated as $15 \%$. In this regard, the essential component of prevention and planning is knowledge of laboratory and demographic findings among COVID-19 patients; therefore, the present study was conducted to investigate laboratory and demographic
\end{abstract}

Correspondence: Neda Mahdavifar, Department of Biostatistics and Epidemiology, School of Health, Non-Communicable Diseases Research Center, Sabzevar University of Medical Sciences, Sabzevar, Iran.

Tel. +98 9158497587. E-mail: n.mahdavifar90@gmail.com

Authors' contribution: All the authors have read and approved the final version of the manuscript and agreed to be accountable for all aspects of the work.

Funding: None.

Conflict of interest: The authors declare no potential conflict of interest.

Ethical approval: Not applicable.

Key words: COVID-19, laboratory finding, systematic reviews.

Received for publication: 23 November 2020.

Accepted for publication: 15 March 2021.

COCopyright: the Author(s), 2021

Licensee PAGEPress, Italy

Monaldi Archives for Chest Disease 2021; 91:1694

doi: 10.4081/monaldi.2021.1694

This article is distributed under the terms of the Creative Commons Attribution Noncommercial License (by-nc 4.0) which permits any noncommercial use, distribution, and reproduction in any medium, provided the original author(s) and source are credited. findings among these patients worldwide. This systematic review was performed on the articles published in English between January 1, 2019 and May 4, 2020, using MeSH-compliant keywords such as "COVID-19", "Laboratory, coronavirus disease-19 testing", and " demography " in international databases (PubMed, and web of science Scopus). Thereafter, the articles relevant to laboratory and demographic findings among COVID-19 patients were included in the final review. Reviewing the included articles showed changes in the mean lymphocytes count ranged from 0.7 to 39 in hospital or severe cases. Moreover, Leukopenia was not observed in patients with thrombocytopenia. In addition, C-reactive protein (CRP), leukocytes, D-dimer, fibrine degradation products (FDP), fibrinogen (FIB), neutrophils, aspartate aminotransferase (AST), serum creatinine, t-troponin, troponin I, and blood bilirubin levels showed increasing trends in most studies conducted on COVID-19 patients. Notably, the elevated lactate dehydrogenase (LDH) level was more common among children than adults. According to the results of the present study, and by considering the clinical characteristics of COVID-19 patients on the one hand, and considering the changes in laboratory samples such as lymphocytes and other blood markers due to the damaged myocardial, hepatic, and renal tissues on the other hand, it is recommended to confirm the diagnosis of this infection by evaluating the patients' blood samples using other diagnostic methods like lung scan.

\section{Introduction}

On December 31, 2019, China notified the World Health Organization (WHO) of a pneumonia of unknown etiology in Wuhan city, China. Afterward, Chinese government reported 440 cases of pneumonia of unknown etiology to WHO from December 31, 2019 to January 3, 2020. Subsequently, WHO received more information from the Chinese National Health Commission about the outbreak that was found to be associated with the exposure to a seafood market in Wuhan city. Correspondingly, Chinese authorities have identified it as a novel strain of coronavirus [1]. Initially, it was stated that the mortality rate of this novel coronavirus (2019$\mathrm{nCoV}$ ) was $15 \%$. According to the results of the subsequent studies, this rate was lower than reported and between 4.3 and $11 \%$ [2, 3]. On March 11, 2020, WHO declared this disease a pandemic [4]. 
The coronaviruses' family can genotypically and serologically be divided into four genera of viruses: alpha, beta, delta, and gamma. The novel coronavirus (2019-nCoV) belongs to the beta-coronavirus category. Furthermore, it is the third known animal coronavirus disease, after SARS and MERS, both of which belong to the beta-coronavirus category [5]. The incubation period of this disease is 14 days, but is usually between 3 and 7 days [6]. Most of the patients with COVID-19 experience mild to moderate symptoms. Notably, it was shown that older people and those with some diseases such as cardiovascular disease, cerebrovascular disease, diabetes, chronic respiratory disease, and cancer are more likely to experience severe symptoms of the disease. Women also are less susceptible to the disease than men. Currently, there is vaccine not available to everyone or no treatment for COVID-19 [2,6,7]. The mean age of the infected patients was between 50 and 69 years old in a Chinese case-series [8]. The disease has also been reported among infants and children, and the latter tend to have milder symptoms compared to adults [9-11]. People's practice regarding home quarantine to prevent COVID-19 depends on a number of factors. Paying attention to the gender, attitude and occupation of individuals in policy making regarding home quarantine can improve the performance of the public [12] Laboratory symptoms are as follows: COVID-19-positive throat, sputum, lower respiratory tract secretion, stool, and blood swab samples for nucleic acid [13]. Additionally, other laboratory symptoms include the elevated erythrocyte sedimentation rate (ESR) by $85 \%$, C-reactive protein (CRP) by $63 \%$, the decreased albumin by $98 \%$, the elevated serum ferritin, the elevated liver function tests, and the elevated lactate dehydrogenase (LDH), the elevated D-Dimer, the elevated blood neutrophils, the elevated interleukin 6 , and the elevated troponin, the decreased blood lymphocytes, the decreased or elevated leukocytes, the decreased or elevated platelets, the impaired coagulation tests, and the decreased hemoglobin [2,14]. Laboratory results vary according to patients' age and disease's severity. It is noteworthy that blood tests are usually normal in children, with transiently high or normal CRP and white blood-cell count (WBC) [10,15].

Considering the increasing incidence of this disease, and since most of the previous studies have been conducted in a specific area with a limited sample size, the essential component of prevention and planning is knowledge of laboratory and demographic findings among COVID-19 patients. Therefore, the present study was conducted to investigate laboratory and demographic findings among the patients with COVID-19 worldwide.

\section{Materials and Methods}

\section{Eligibility criteria}

The present narrative review study was performed on full-text original English articles published in foreign journals from January 1, 2019 to May 4, 2020.

\section{Information sources}

Articles were selected from databases (PubMed, Web of Science and Scopus).

\section{Search}

\section{Study selection}

Those articles related to laboratory and demographic findings among COVID-19 patients were reviewed. The results of these studies are presented in the tables. It should be noted that we excluded unrelated studies.

\section{Data collection process}

Summary and full text of the included articles were reviewed by two relevant researchers independently. Moreover, the checklist information including author's name, year of publication, place of study, sample size, demographic characteristics (age and sex), and laboratory findings were recorded.

\section{Data items}

Articles were selected from international databases using all MeSH-compliant keywords including "COVID-19", "Laboratory, Coronavirus disease-19 testing", and "Demography".

\section{Summary measures}

The articles conducted on demographic characteristics and laboratory findings from January 1, 2019 to May 4, 2020, were selected and the results were presented in tabular form.

\section{Synthesis of results}

A total of 2675 articles were initially reviewed. It should be noted that 675 articles were duplicates, 800 articles were fully read, and 740 articles were irrelevant, so 60 articles were finally included in the study (Figure 1).

\section{Results}

SARS-CoV-2 is an emerging pathogen that caused a high incidence of pneumonia in the infected individual [16]. Understanding the clinical and laboratory characteristics of patients will be helpful in diagnosis and treatment of this disease.

\section{Lymphocytes and cytokines}

In this study, the mean lymphocyte counts in hospital or in more severe cases ranged from 0.7 to 39 (Table 1). Changes in lymphocyte count may be related either to the severity or to the prognosis of COVID-19 [17]. Lymphocytopenia commonly occurs in patients with SARS-CoV-2 [18]. In addition, T lymphocytes act as antivirus and balance immune responses. CD8p T cells play a vital role in SARS-CoV virus killing in the patients' lungs [19,20]. IL-6 is also known as a key cytokine that plays an important role in host defense by stimulating the immune response, bleeding, and immune responses. It should be noted that IL-6 levels dramatically increase during infection $[21,22]$. The results of studies conducted on SARS-CoV indicated that the reduction of CD4p T cells plays a role in reducing lymphocyte uptake and cytokine production, which also has a negative effect on improving SARS-CoV symptoms [23]. Cytokines play important roles in viral infections. In fact, cytokine level significantly increases in patients with more severe disease [24].

\section{White blood-cell count, D-dimer, fibrine degradation products, fibrinogen, and C-reactive protein}

In the present study, except in patients with thrombocytopenia, leukopenia (white blood cell (WBC) count $<4 \times 109 /$ L) was not observed in the patients (Table 1). In other studies, it was found that the elevated WBC count occurs more in children than in adults, which indicates leukopenia in most cases, while lymphocyte count decreases in adults [25]. The results show that D-dimer, 
FDP, and FIB have increased in COVID-19 patients in most studies, which is somewhat consistent with the results of the other relevant studies [26,27]. The CRP level has also increased in most studies. It was shown that the elevated CRP levels also occur more frequently than alanine aminotransferase (ALT), AST, creatine kinaser, and D-dimer (Table 1), which is consistent with the findings of the other studies conducted on patients with MERS-CoV and SARS-CoV [28-30].

\section{Serum creatinine, troponin T, troponin I, blood bilirubin, lactate dehydrogenase leukocytes, and neutrophils}

In the present study, the findings demonstrated an increase in AST, serum creatinine, troponin T, troponin I, and blood bilirubin, which indicate damages to myocardial, liver, and kidney of the COVID-19 patients. Accordingly, elevated AST, ALT, and $\gamma$-GT levels among COVID-19 patients are often associated with liver damage [31]. Moreover, the decreased lymphocytes and the elevated CRP, IL-6, ESR, and d-dimer can be associated with liver, kidney, and myocardial damages as well [32]. The elevated blood urea and creatinine levels have been reported in the blood of patients before death [17]. In a study by Du et al., it was found that more than half of COVID-19 patients experience impaired renal function [33], due to the elevated blood urea and creatinine levels. The increased LDH could also indicate the fact that SARS-CoV-2 infection is associated with cardiopulmonary tissue damage among children. Consistently with the other studies, the results of the present study show the elevated LDH in children compared to adults. The elevated LDH and blood sugar levels are two important factors in the diagnosis of COVID-19 patients [34]. On the other hand, reduc- tion of CD8 T cells and loss of eosinophils due to SARS-CoV-2 are effective on causing eosinopenia and eventual death resulted from chronic respiratory diseases, because IL-5 produced by CD8 T cells helps in proliferating and activating eosinophils $[35,36]$. The results of the present study show an increase in leukocyte and neutrophil levels. The increased neutrophil counts were also observed in severe pneumonia caused by COVID-19 [37]. In fact, it can be said that laboratory changes in patients indicate the progression of the disease. Therefore, it is very important to perform the relevant tests on COVID-19 patients.

\section{Discussion}

Blood biochemical indicators such as C-reactive protein, lymphocytes, AST, ALT neutrophils, FDP and FIB, D-dimer, LDH, Creactive protein are the best predictors of disease progression in patients with Covid-19 [38]. Coagulation is a physiological response to infections 30,39-41]. In patients, coagulation increases excessively due to increased levels of D-dimer, FDP, and FIB [42].

In addition, studies showed that the coronavirus can induce the body to produce oxidative stress and release a large amount of active oxygen free radicals, which can, on one hand, make the virus replicate unceasingly, but, on the other hand, the excessive free radicals can damage the body's biological membrane lipid peroxidation, enzyme, amino acid, and oxidative protein, leading to injury to the organs, such as the lung, heart, liver, kidney [43].

One of the limitations of the present study is the lack of sys-

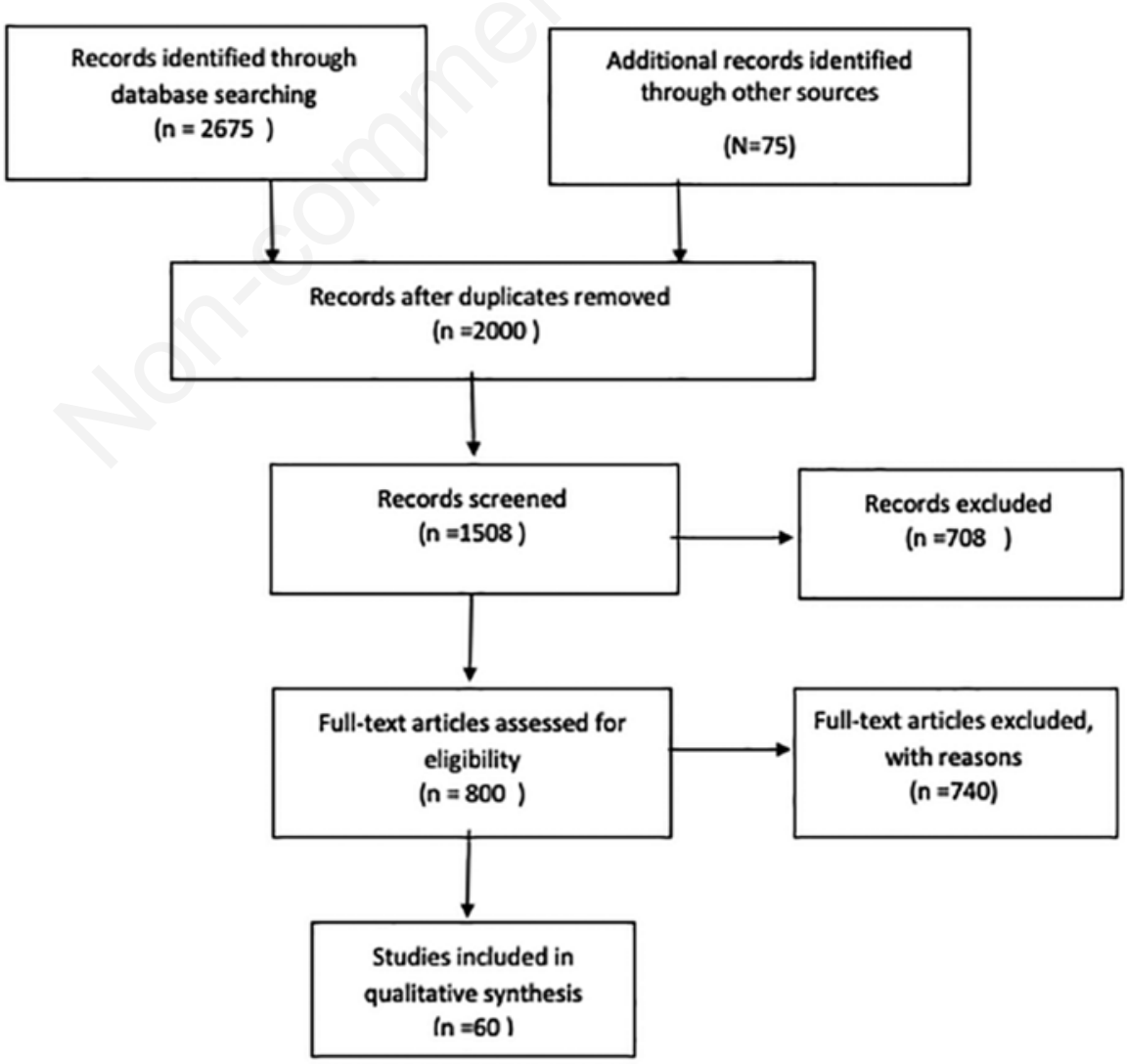

Figure 1. Flowchart of articles included in this review. 
tematic reviews of articles that have simultaneously reported lung scan information of patients in order to provide more comprehensive information to researchers.

\section{Conclusions}

According to the results of the present study, considering the clinical characteristics of COVID-19 patients on the one hand, and changes in laboratory samples such as lymphocytes and other blood markers due to damages to myocardial, hepatic, and renal tissues on the other hand, it is recommended to confirm the diagnosis of this infection by evaluating the patients' blood samples using other diagnostic methods like lung scan.

\section{References}

1. World Health Organization. Novel Coronavirus (2019-nCoV) Situation Report. Accessed: 21 January 2020.

2. Chen NS, Zhou M, Dong X, et al. Epidemiological and clinical characteristics of 99 cases of 2019 novel coronavirus pneumonia in Wuhan, China: a descriptive study. Lancet 2020;395:507-13.

3. Yang WJ, Cao QQ, Qin L, et al. Clinical characteristics and imaging manifestations of the 2019 novel coronavirus disease (COVID-19):A multi-center study in Wenzhou city, Zhejiang, China. J Infect 2020;80:388-93.

4. Ramphul K, Mejias SG. Coronavirus disease: A review of a new threat to public health. Cureus 2020;12:e7276.

5. Chen Y, Liu Q, Guo D. Emerging coronaviruses: Genome structure, replication, and pathogenesis. J Med Virol 2020;92:418-23.

6. Zhou M, Zhang X, Qu J. Coronavirus disease 2019 (COVID19): a clinical update. Front Med 2020;14:126-35.

7. World Health Organization. Coronavirus 2020. Available from: https://www.who.int/healthtopics/coronavirus\#tab=tab_1

8. The Novel Coronavirus Pneumonia Emergency Response Epidemiology Team. The Epidemiological characteristics of an outbreak of 2019 novel coronavirus diseases (COVID-19) China, 2020. China CDC Weekly 2020;8:113-22.

9. Singhal T. A review of coronavirus disease-2019 (COVID-19). Indian J Pediatr 2020;87:281-6.

10. Shen KL, Yang YH. Diagnosis and treatment of 2019 novel coronavirus infection in children: a pressing issue. World $\mathrm{J}$ Pediatr 2020;16:219-21.

11. Xia W, Shao J, Guo Y, et al. Clinical and CT features in pediatric patients with COVID-19 infection: Different points from adults. Pediatr Pulmonol 2020;55:1169-74.

12. Fallahi A, Mahdavifar N, Ghorbani A, et al. Public Knowledge, attitude and practice regarding home quarantine to prevent COVID-19 in Sabzevar city, Iran. J Mil Med 2020;22:580-8.

13. Shen KL, Yang YH, Wang TY, et al. Diagnosis, treatment, and prevention of 2019 novel coronavirus infection in children: experts' consensus statement. World J Pediatr 2020;16:223-31.

14. Lake MA. What we know so far: COVID-19 current clinical knowledge and research. Clin Med 2020;20:124-7.

15. Pan L, Mu M, Yang P, et al. Clinical characteristics of COVID19 patients with digestive symptoms in Hubei, China: A descriptive, cross-sectional, multicenter study. Am J Gastroenterol 2020;115:766-73.
16. Qiu H, Wu J, Hong L, et al. Clinical and epidemiological features of 36 children with coronavirus disease 2019 (COVID19) in Zhejiang, China: an observational cohort study. Lancet Infect Dis 2020;20:689-96.

17. Wang D, Hu B, Hu C, et al. Clinical characteristics of 138 hospitalized patients with 2019 novel coronavirus-infected pneumonia in Wuhan, China. JAMA 2020;323:1061-9.

18. Du Y, Tu L, Zhu P, et al. Clinical features of 85 fatal cases of COVID-19 from Wuhan. A retrospective observational study. Am J Respir Crit Care Med 2020;201:1372-9.

19. Cecere TE, Todd SM, LeRoith T. Regulatory $\mathrm{T}$ cells in arterivirus and coronavirus infections: do they protect against disease or enhance it? Viruses 2012;4:833-46.

20. Channappanavar R, Fett C, Zhao J, Meyerholz DK, Perlman S. Virus-specific memory CD8 $\mathrm{T}$ cells provide substantial protection from lethal severe acute respiratory syndrome coronavirus infection. J Virol 2014;88:11034-44.

21. Tanaka T, Narazaki M, Kishimoto T. IL-6 in inflammation, immunity, and disease. Cold Spring Harb Perspect Biol 2014;6:a016295.

22. Narazaki M, Kishimoto T. The two-faced cytokine IL-6 in host defense and diseases. Int J Mol Sci 2018;19:3528.

23. Chen J, Lau YF, Lamirande EW, et al. Cellular immune responses to severe acute respiratory syndrome coronavirus (SARS-CoV) infection in senescent BALB/c mice: CD4+T cells are important in control of SARS-CoV infection. J Virol 2010;84:1289-301.

24. Wang R, Pan M, Zhang X, et al. Epidemiological and clinical features of 125 Hospitalized Patients with COVID-19 in Fuyang, Anhui, China. Int J Infect Dis 2020;95:421-8.

25. Han YN, Feng ZW, Sun LN, et al. A comparative-descriptive analysis of clinical characteristics in 2019-coronavirus-infected children and adults. J Med Virol 2020;92:1596-602.

26. Tang N, Li D, Wang X, Sun Z. Abnormal coagulation parameters are associated with poor prognosis in patients with novel coronavirus pneumonia. J Thrombs Haemost 2020;18:844-7.

27. Han H, Yang L, Liu R, et al. Prominent changes in blood coagulation of patients with SARS-CoV-2 infection. Clin Chem Lab Med 2020;58:1116-20.

28. Zhang G, Hu C, Luo L, et al. Clinical features and short-term outcomes of 221 patients with COVID-19 in Wuhan, China. J Clin Virol 2020;127:104364.

29. Chen N, Zhou M, Dong X, et al. Epidemiological and clinical characteristics of 99 cases of 2019 novel coronavirus pneumonia in Wuhan, China: a descriptive study. Lancet 2020;395:507-13.

30. Huang C, Wang Y, Li X, et al. Clinical features of patients infected with 2019 novel coronavirus in Wuhan, China. Lancet 2020;395:497-506.

31. Zhao D, Yao F, Wang L, et al. A comparative study on the clinical features of COVID-19 pneumonia to other pneumonias. Clin Infect Dis 2020;71:756-61.

32. Cai Q, Huang D, Ou P, et al. COVID-19 in a designated infectious diseases hospital outside Hubei Province, China. Allergy 2020;75:1742-52.

33. Du Y, Tu L, Zhu P, et al. Clinical features of 85 fatal cases of COVID-19 from Wuhan: A retrospective observational study. Am J Respir Crit Care Med 2020;201:1372-9.

34. Jin X, Lian J-S, Hu J-H, Gao J, et al. Epidemiological, clinical and virological characteristics of 74 cases of coronavirusinfected disease 2019 (COVID-19) with gastrointestinal symptoms. Gut 2020;69:1002-9.

35. Liu Y, Yang Y, Zhang C, et al. Clinical and biochemical indexes 
from 2019-nCoV infected patients linked to viral loads and lung injury. Sci China Life Sci 2020;63):364-74.

36. Schwarze J, Cieslewicz G, Joetham A, et al. CD8 T cells are essential in the development of respiratory syncytial virusinduced lung eosinophilia and airway hyperresponsiveness. J Immunol 1999;162:4207-11.

37. Zhang G, Zhang J, Wang B, et al. Analysis of clinical characteristics and laboratory findings of 95 cases of 2019 novel coronavirus pneumonia in Wuhan, China: a retrospective analysis. Respir Res. 2020;21:74.

38. Cao J, Tu WJ, Cheng W, et al. Clinical features and short-term outcomes of 102 patients with coronavirus disease 2019 in Wuhan, China. Clin Infect Dis 2020;71:748-55.

39. Chen J, Qi T, Liu L, et al. Clinical progression of patients with COVID-19 in Shanghai, China. J Infect 2020;80:e1-e6.

40. Chen J, Zhang ZZ, Chen YK, et al. The clinical and immunological features of pediatric COVID-19 patients in China. Genes Dis 2020;7:535-41.

41. Chen N, Zhou M, Dong X, et al. Epidemiological and clinical characteristics of 99 cases of 2019 novel coronavirus pneumonia in Wuhan, China: a descriptive study. Lancet 2020;395:507-13.

42. Chen Q, Zheng Z, Zhang C, et al. Clinical characteristics of 145 patients with corona virus disease 2019 (COVID-19) in Taizhou, Zhejiang, China. Infection 2020;48:543-51.

43. Chen T, Dai Z, Mo P, et al. Clinical characteristics and outcomes of older patients with coronavirus disease 2019 (COVID-19) in Wuhan, China (2019): a single-centered, retrospective study. J Gerontol A Biol Sci Med Sci 2020;75:1788-95.

44. Chen X, Tang Y, Mo Y, et al. A diagnostic model for coronavirus disease 2019 (COVID-19) based on radiological semantic and clinical features: a multi-center study. Eur Radiol 2020;30:4893-902.

45. Chen X, Zhao B, Qu Y, et al. Detectable serum SARS-CoV-2 viral load (RNAaemia) is closely correlated with drastically elevated interleukin 6 (IL-6) level in critically ill COVID-19 patients. Clin Infect Dis 2020;71:1937-42.

46. Chen Y, Chen L, Deng Q, et al. The presence of SARS-CoV-2 RNA in the feces of COVID-19 patients. J Med Virol 2020;92:833-40.

47. Cheng Z, Lu Y, Cao Q, et al. Clinical features and chest CT manifestations of coronavirus disease 2019 (COVID-19) in a single-center study in Shanghai, China. AJR Am J Roentgenol 2020;215:121-6.

48. Deng Y, Liu W, Liu K, et al. Clinical characteristics of fatal and recovered cases of coronavirus disease 2019 (COVID-19) in Wuhan, China: a retrospective study. Chin Med J (Engl) 2020;133:1261-7.

49. Ding X, Xu J, Zhou J, Long Q. Chest CT findings of COVID19 pneumonia by duration of symptoms. Eur J Radiol 2020;127:109009.

50. Du W, Yu J, Wang H, et al. Clinical characteristics of COVID19 in children compared with adults in Shandong Province, China. Infection 2020;48:445-52.

51. Fan Z, Chen L, Li J, et al. Clinical features of COVID-19-related liver damage. Clin Gastroenterol Hepatol 2020;18:1561-6.

52. Gao Y, Li T, Han M, et al. Diagnostic utility of clinical laboratory data determinations for patients with the severe COVID19. J Med Virol 2020;92:791-6.

53. Guan WJ, Ni ZY, Hu Y, et al. Clinical characteristics of coronavirus disease 2019 in China. N Engl J Med 2020;382:1708-20.

54. Han H, Yang L, Liu R, et al. Prominent changes in blood coagulation of patients with SARS-CoV-2 infection. Clin Chem Lab Med 2020;58:1116-20.
55. Jin X, Lian JS, Hu JH, et al. Epidemiological, clinical and virological characteristics of 74 cases of coronavirus-infected disease 2019 (COVID-19) with gastrointestinal symptoms. Gut 2020;69:1002-9.

56. Li B, Shen J, Li L, Yu C. Radiographic and clinical features of children with 2019 novel coronavirus (COVID-19) pneumonia. Indian Pediatr 2020;57:423-6.

57. Li J, Wang X, Chen J, et al. COVID-19 infection may cause ketosis and ketoacidosis. Diabetes Obes Metab 2020;22:1935-41.

58. Li K, Wu J, Wu F, et al. The clinical and chest CT features associated with severe and critical COVID-19 pneumonia. Invest Radiol 2020;55:327-31.

59. Li X, Zeng W, Li X, et al. CT imaging changes of corona virus disease 2019(COVID-19): a multi-center study in Southwest China. J Transl Med. 2020;18:154.

60. Liu Y, Sun W, Guo Y, et al. Association between platelet parameters and mortality in coronavirus disease 2019: Retrospective cohort study. Platelets 2020;31:490-6.

61. Mao L, Jin H, Wang M, et al. Neurologic manifestations of hospitalized patients with coronavirus disease 2019 in Wuhan, China. JAMA Neurol 2020;77:68-90.

62. Meng H, Xiong R, He R, et al. CT imaging and clinical course of asymptomatic cases with COVID-19 pneumonia at admission in Wuhan, China. J Infect 2020;81:e33-9.

63. Meng Y, Wu P, Lu W, et al. Sex-specific clinical characteristics and prognosis of coronavirus disease-19 infection in Wuhan, China: A retrospective study of 168 severe patients. PLoS Pathog 2020;16:e1008520.

64. Qian GQ, Yang NB, Ding F, et al. Epidemiologic and clinical characteristics of 91 hospitalized patients with COVID-19 in Zhejiang, China: A retrospective, multi-centre case series. QJM 2020;113:474-81.

65. Shi H, Han X, Jiang N, et al. Radiological findings from 81 patients with COVID-19 pneumonia in Wuhan, China: a descriptive study. Lancet Infect Dis 2020;20:425-34.

66. To KK, Tsang OT, Leung WS, et al. Temporal profiles of viral load in posterior oropharyngeal saliva samples and serum antibody responses during infection by SARSCoV-2: an observational cohort study. Lancet Infect Dis 2020;20: 565-74.

67. Wan S, Xiang Y, Fang W, et al. Clinical features and treatment of COVID-19 patients in northeast Chongqing. J Med Virol 2020;92:797-806.

68. Wang L, Duan Y, Zhang W, Liang J, Xu J, Zhang Y, et al. Epidemiologic and clinical characteristics of 26 cases of COVID-19 arising from patient-to-patient transmission in Liaocheng, China. Clin Epidemiol 2020;12:387-91.

69. Wang R, Pan M, Zhang X, et al. Epidemiological and clinical features of 125 hospitalized patients with COVID-19 in Fuyang, Anhui, China. Int J Infect Dis 2020;95:421-8.

70. Wang X, Liu W, Zhao J, et al. Clinical characteristics of 80 hospitalized frontline medical workers infected with COVID-19 in Wuhan, China. J Hosp Infect 2020;105:399-403.

71. Wang Z, Yang B, Li Q, Wen L, Zhang R. Clinical features of 69 cases with coronavirus disease 2019 in Wuhan, China. Clin Infect Dis 2020;71:769-77.

72. Wu J, Liu J, Zhao X, et al. Clinical characteristics of imported cases of COVID-19 in Jiangsu Province: A multicenter descriptive study. Clin Infect Dis 2020;71:706-12.

73. Wu P, Duan F, Luo C, et al. Characteristics of ocular findings of patients with coronavirus disease 2019 (COVID-19) in Hubei Province, China. JAMA Ohthalmol Clin Infect Dis 2020;71:706-12. 
74. Xia W, Shao J, Guo Y, et al. Clinical and CT features in pediatric patients with COVID-19 infection: Different points from adults. Pediatr Pulmonol 2020;55:1169-74.

75. Xie H, Zhao J, Lian N, et al. Clinical characteristics of nonICU hospitalized patients with coronavirus disease 2019 and liver injury: A retrospective study. Liver Int 2020;40:1321-6.

76. Xiong Y, Sun D, Liu Y, et al. Clinical and High-resolution CT features of the COVID-19 infection: Comparison of the initial and follow-up changes. Invest Radiol 2020;55:332-9.

77. Young BE, Ong SWX, Kalimuddin S, et al. Epidemiologic features and clinical course of patients infected with SARS-CoV2 in Singapore. JAMA 2020;323:1488-94.

78. Zhang J, Liu P, Wang M, et al. The clinical data from 19 critically ill patients with coronavirus disease 2019: a single-centered, retrospective, observational study. Z Gesundh Wiss 2020;1-4. Online ahead of print.

79. Zhang R, Ouyang H, Fu L, et al. CT features of SARS-CoV-2 pneumonia according to clinical presentation: a retrospective analysis of 120 consecutive patients from Wuhan city. Eur Radiol 2020;30:4417-26.
80. Zhang X, Cai H, Hu J, et al. Epidemiological, clinical characteristics of cases of SARSCoV-2 infection with abnormal imaging findings. Int J Infect Dis 2020;94:81-7.

81. Zhao X, Liu B, Yu Y, et al. The characteristics and clinical value of chest CT images of novel coronavirus pneumonia. Clin Radiol 2020;75:335-40.

82. Zheng F, Liao C, Fan QH, et al. Clinical characteristics of children with coronavirus disease 2019 in Hubei, China. Curr Med Sci 2020;40:275-80.

83. Zheng F, Tang W, Li H, et al. Clinical characteristics of 161 cases of corona virus disease 2019 (COVID-19) in Changsha. Eur Rev Med Pharmacol Sci 2020;24:3404-10.

84. Zheng Y, Xu H, Yang M, et al. Epidemiological characteristics and clinical features of 32 critical and 67 noncritical cases of COVID-19 in Chengdu. J Clin Virol 2020;127:104366.

85. Zhou Z, Guo D, Li C, et al. Coronavirus disease 2019: initial chest CT findings. Eur Radiol 2020;30:4398-406.

86. Zhu W, Xie K, Lu H, et al. Initial clinical features of suspected coronavirus disease 2019 in two emergency departments outside of Hubei, China. J Med Virol 2020;92:1525-32. 